\title{
Lagrangian Heuristics Based on Disaggregated Bundle Methods for Hydrothermal Unit Commitment
}

\author{
A. Borghetti, A. Frangioni, F. Lacalandra, C. A. Nucci
}

\begin{abstract}
The paper presents a simple and effective Lagrangian relaxation approach for the solution of the optimal short-term unit commitment problem in hydrothermal power-generation systems. The proposed approach, based on a disaggregated Bundle method for the solution of the dual problem, with a new warm-starting procedure, achieves accurate solutions in few iterations. The adoption of a disaggregated Bundle method not only improves the convergence of the proposed approach but also provides information that are suitably exploited for generating a feasible solution of the primal problem and for obtaining an optimal hydro scheduling. A comparison between the proposed Lagrangian approach and other ones, based on sub-gradient and Bundle methods, is presented for a simple yet reasonable formulation of the Hydrothermal Unit Commitment problem.
\end{abstract}

Index Terms-Power generation operation, Hydrothermal unit commitment, Power generation dispatch, Lagrangian Relaxation, Bundle methods.

\section{NOMENCLATURE}

$I$

$B$

$H, H_{b}$

$H_{h}^{\prime}$

$T$

D

$\mathbf{R}$

set of indexes of available thermal units $(|I|$ : number of thermal units; $i$ : thermal unit index). set of indexes of river basins $(|B|$ : number of river basins; $b$ : basin unit index).

set of indexes of all available hydro units and those in river basin $b$, respectively, $(|H|$ : number of hydro units, $\left|H_{b}\right|$ : number of hydro units in river basin $b$; $h$ : hydro unit index).

set of indexes of available upstream hydro units in river basin $b$ directly above hydro unit $h$ (|$H_{h}^{\prime} \mid$ : number of hydro units; $h^{\prime}$ : upstream hydro unit index).

set of time periods in the optimization horizon ( $|T|$ : number of time periods; $t$ : time period index).

D $|T|$-dimensional vector of load demands $D_{t}$ in each period $t$.

$\mathbf{R} \quad|T|$-dimensional vector of the required operating

This work was supported in part by the Italian National Research Council under Grant CNRC00C821.

A. Borghetti, F. Lacalandra, C. A. Nucci are with the Department of Electrical Engineering, University of Bologna, 40136 Bologna, Italy.

A. Frangioni is with the Department of Computer Science, University of Pisa, 56125 Pisa, Italy. $\mathbf{u}$

spinning reserves $R_{t}$ in each period $t$.

$|I|$-rows $|T|$-columns matrix, whose rows are the $|T|$-dimensional arrays $\mathbf{u}_{i}$ of the $0-1$ variables $u_{i, t}$ indicating the commitment state of thermal unit $i$ during period $t$.

$\mathbf{p}_{I} \quad|I|$-rows $|T|$-columns matrix, whose rows are the $|T|$-dimensional arrays $\mathbf{p}_{i}$ of production levels $p_{i, t}$ of thermal unit $i$ during each period $t$.

$\mathbf{p}_{H} \quad|H|$-rows $|T|$-columns matrix, whose rows are the $|T|$-dimensional arrays $\mathbf{p}_{h}$ of production levels $p_{h, t}$ of hydro unit $h$ during each period $t$.

$p_{i}, \bar{p}_{i}, \bar{p}_{h}$ minimum/maximum outputs of the units.

$\bar{\tau}_{i}^{u}, \tau_{i}^{d} \quad$ minimum up- and down-times of thermal units.

$c_{i, t} \quad$ per hour operating cost of committed thermal unit $i$ at period $t$, as a quadratic function of production level $p_{i, t}$.

$r_{i, t}, r_{h, t} \quad$ operating reserve contributions that can be supplied by the units during period $t$ as a function of their production levels.

$s_{i, t}^{u} \quad$ start-up cost which is charged whenever thermal unit $i$ is committed at the beginning of period $t$. It can depend on the number of periods that the unit has been down.

$w_{h, t}, a_{h, t}, s_{h, t}$ water discharge rate, net inflow rate and spillage of reservoir $h$ during period $t$.

$n_{t} \quad$ length of period $t$. Summation $\sum_{t \in T} n_{t}$ is equal to the optimization horizon.

$\tau_{h^{\prime}, h} \quad$ water transport delay from hydro unit $h^{\prime}$ to reservoir $h$.

$V_{h, t} \quad$ storage volume of the reservoir of hydro unit $h$ at the end of period $t$, limited between a maximum $\bar{V}_{h}$ and a minimum $\underline{V}_{h}$ value.

$V_{h \text {,in }}, V_{h \text {,end }}$ storage volumes of reservoir $h$ at the beginning and at the end of the optimization horizon, as given by a long-term hydro-scheduling.

\section{INTRODUCTION}

$\mathrm{T}$ HIS paper deals with the solution of short-term unit commitment (UC) problems in hydrothermal power-generation systems. Obtaining good schedules of electric power generating units over a daily to weekly time horizon can considerably reduce the production costs, which is 
of increasing importance in the ongoing liberalization of the electricity market in many countries [1].

The optimization model dealt with in this paper takes into account the main operating constraints and physical characteristics of a hydrothermal power generation system. The relevant mathematical formulation consists of a large-scale mixed-integer non-linear optimization problem (e.g. [2]).

The solution approach that is most widely used at present is Lagrangian relaxation (e.g. [3-24]), due also to its ability to include more detailed system representation than would be possible with other techniques [1]. In the Lagrangian relaxation (LR) approach, the problem is decomposed into independent single-unit problems by relaxing the coupling constraints, and the corresponding (Lagrangian) dual problem is solved seeking for the optimal multipliers of the relaxed constraints. Heuristics are then used to obtain a feasible schedule.

Different non-differentiable optimization methods have been proposed for the solution of the Lagrangian dual. The correct choice of the method is critical both for the efficiency of the lower bound computation and for the quality of the primal solution obtained. The first approaches have used sub-gradient methods (e.g. [3-6]) that require low computing effort per iteration, but have also low convergence characteristics. Recently, LR approaches have been presented based on dynamically constrained [7] and Interior-Point Cutting-Plane methods [8], and Bundle methods in different variants: aggregated, disaggregated (e.g. [9-16]) and reduced-complexity ones [17]. In order to overcome the non-differentiability of the Lagrangian function, Augmented Lagrangian (AL) approaches (e.g. [18-21]) have also been used. In this case, however, the AL is not directly separable between production units. It is well known (e.g. $[25,26])$ that generalized Bundle methods are just approximated Augmented Lagrangians.

This paper presents a simple yet effective LR approach for the hydrothermal UC problem, fitted by a Lagrangian heuristic that exploits the information provided by solving the dual problem with a proximal Bundle method, and by a "warm-starting" procedure that improves both convergence and quality of the solutions. One of the main points of the proposed approach is the adoption of a disaggregated bundle method. The aim of this paper is to show the influence of the Lagrangian optimization procedure on the performance of the Lagrangian heuristic. In particular, we underline the importance of using disaggregated methods and of exploiting the available primal information.

In the next section, Section III, a mathematical formulation of the problem is given. This is followed, in Section IV, by a description of the LR approach and of the solution of the dual problem by aggregated and disaggregated Bundle methods. In Section $\mathrm{V}$ the proposed Lagrangian heuristics and warm-starting procedure for hydrothermal UC are presented. In Section VI, by means of computational results, we examine the impact of the proposed heuristics both on the convergence characteristics of aggregated and disaggregated Bundle methods for solving the dual problem, and on the quality of the obtained UC.

\section{PROBLEM FORMULATION}

Modeling of UC is very diverse, due to the different types of thermal generation (conventional, nuclear, combined-cycle, heat and power cogeneration, etc.) and of hydro generation (with pump-storage plants that can be hydraulically coupled and subjected to very different natural and manmade constraints), to the presence of transmission constraints, emission constraints, etc [27].

The present analysis of the behavior of the proposed Lagrangian heuristics has been carried out on the following formulation of the hydrothermal UC problem, which takes into account the main operating constraints and the physical characteristics of the power generation system, usually considered for the problem of interest (e.g. [2]). In particular, we consider a power system of $|T|$ thermal units and $|B|$ river basins, each with $\left|H_{b}\right|$ hydro units. The aim is to determine the hydrothermal UC that minimizes the sum of operating $\operatorname{costs} c_{i, t}$ and start-up costs $s_{i, t}^{u}$ of committed thermal units

$$
\min _{\mathbf{u}, \mathbf{p}_{I}, \mathbf{p}_{H}} \sum_{i \in I} \sum_{t \in T}\left[u_{i, t} \cdot c_{i, t}\left(p_{i, t}\right)+s_{i, t}^{u}\left(\mathbf{u}_{i}\right)\right]=\min _{\mathbf{u}, \mathbf{p}_{I}, \mathbf{p}_{H}} C\left(\mathbf{u}, \mathbf{p}_{I}\right)
$$

and satisfies the forecasted demands $\mathbf{D}$ and operating spinning reserve $\mathbf{R}^{1}$ (global constraints),

$$
\begin{array}{ll}
\sum_{i \in I} u_{i, t} \cdot p_{i, t}+\sum_{h \in H} p_{h, t}=D_{t} & \forall t \in T \\
\sum_{i \in I} u_{i, t} \cdot r_{i, t}+\sum_{h \in H} r_{h, t} \geq R_{t} & \forall t \in T
\end{array}
$$

without violating physical and operating constraints of the generation units, disregarding network and emission constraints. The considered operating constraints for each thermal unit $i$ and time period $t$ are

$$
\begin{aligned}
& u_{i, t} \cdot \underline{p}_{i} \leq p_{i, t} \leq u_{i, t} \cdot \bar{p}_{i} \\
& \tau_{i}^{d} \text { and } \tau_{i}^{u} \text { constraints. }
\end{aligned}
$$

If the time step is chosen particularly small with comparison of the slow dynamical response of some thermal power plants, the outputs of slow unit $i$ is limited by the socalled ramp-rate constraints:

$\Delta_{i}^{d} \leq p_{i, t} \cdot u_{i, t}-p_{i, t-1} \cdot u_{i, t} \leq \Delta_{i}^{u} \quad \forall t \in T$

where $\Delta_{i}^{d}$ and $\Delta_{i}^{u}$ are the maximum decrease and increase, respectively, in the output of unit $i$ in one time period. Taking into account these constraints within the Lagrangian framework can be done, by suitably modifying the solution of the thermal generator subproblem, as suggested in $[4,36]$. Alternatively, in [14] the adoption of a "system ramp multiplier" is proposed, whose updating can be carried out,

\footnotetext{
1 Spinning reserve is the difference between the maximum output of all running units and the current output. However, some of this reserve can be non-available within the required time frame due to ramping constraints. The part of spinning reserve that is available is called the operating spinning reserve [27]. For a unit $k$, the operating spinning reserve $r_{k, t}$ can be expressed as [4]

$r_{k, t}=\min \left(p_{k, t}+\Delta_{k}^{\varepsilon}, \bar{p}_{k}\right)-p_{k, t}$

where $\Delta_{i}^{\varepsilon}$ is the maximum amount of emergency power that can be provided within the required short interval of time.
} 
e.g. with bundle methods. Frequently disregarded (e.g. $[5,6,10,13,19,24])^{2}$, these constraints do not affect the exposition and development of our approach and, for the sake of simplicity, are not considered in the following.

The generation of hydroelectric power plants reduces the power demand that must be fulfilled by the costly thermal production. The electrical output of a hydro power plant depends on the water discharge, the head (which can be expressed as a function of the reservoir volume) and the efficiency of the hydraulic turbine (which is also a function of the water discharge and the head). The operating points are restricted by minimal and maximal water discharges. In paragraph V.A a Lagrangian heuristic is proposed that results to be particularly efficient when $p_{h, t}$ is assumed to be a linear function of water discharge $w_{h, t}$ and independent of the water head, assumption frequently considered reasonable in the literature on the subject (e.g. $[2,13,23,24])$. Hydro production is represented by following simplified model that, for each hydro unit $h$ of reservoir network $b$ and for each time period $t$, takes into account the hydraulic continuity equations to compute the reservoir storages, the storage, discharge rate and spillage limits, and the initial and ending storage constraints:

$V_{h, t}-V_{h, t-1}=n_{t} \cdot\left[\left(a_{h, t}-w_{h, t}-s_{h, t}\right)+\sum_{h^{\prime} \in H_{h}^{\prime}}\left(s_{h^{\prime}, t-\tau_{h^{\prime}, h}}+w_{h^{\prime}, t-\tau_{h^{\prime}, h}}\right)\right]$

$\underline{V}_{h} \leq V_{h, t} \leq \bar{V}_{h}$

$\underline{S}_{h} \leq s_{h, t} \leq \bar{S}_{h}$

$0 \leq w_{h, t} \leq \bar{W}_{h}$

$V_{h, \text { in }}$ and $V_{h, \text { end }}$ constraints

The model described by (6) contains an energy constraint since the energy produced by the hydro plants in basin $b$ at the end of the optimization horizon is limited by the total amount of available water to be discharged. This is of interest because, as already observed in the literature $[18,23,24]$ and further discussed in section $\mathrm{V}$ of this paper, energy constraints are one of the main reasons of oscillating behavior of traditional Lagrangian relaxation algorithms.

\section{SOLUTION METHODOLOGY}

\section{A. Lagrangian relaxation approach}

By relaxing the system demand and spinning reserve requirements, (2) and (3) respectively, through Lagrangian multipliers $\lambda_{D_{t}}, \lambda_{R_{t}}$, the following dual function is obtained:

$$
L\left(\boldsymbol{\lambda}_{D}, \boldsymbol{\lambda}_{R}\right)=\min _{\mathbf{u}, \mathbf{p}_{I}, \mathbf{p}_{H}}\left\{\begin{array}{l}
C+\sum_{t \in T} \lambda_{D t} \cdot\left(D_{t}-\sum_{i \in I} u_{i, t} \cdot p_{i, t}-\sum_{b \in B} \sum_{h \in H_{b}} p_{h, t}\right) \\
+\sum_{t \in T} \lambda_{R t} \cdot\left[R_{t}-\sum_{i \in I} u_{i, t} \cdot r_{i, t}-\sum_{b \in B} \sum_{h \in H_{b}} r_{h, t}\right]
\end{array}\right\}
$$

subject to constraints (4) and (6). Dual function (7) exhibits a disaggregated structure:

\footnotetext{
${ }^{2}$ As mentioned in [27], for modern units and UC problems with a one-hour time step, these constraints are not of concern since these units can cross the entire operating domain within much less than one time step.
}

$$
\begin{aligned}
L\left(\lambda_{D}, \lambda_{R}\right) & =\sum_{i \in I} L_{i}\left(\lambda_{D}, \lambda_{R}\right)+\sum_{b \in B} L_{b}\left(\lambda_{D}, \lambda_{R}\right) \\
& +\sum_{t \in T}\left(\lambda_{D_{t}} \cdot D_{t}+\lambda_{R_{t}} \cdot R_{t}\right)
\end{aligned}
$$

where, for thermal unit $i$

$L_{i}\left(\boldsymbol{\lambda}_{D}, \boldsymbol{\lambda}_{R}\right)=\min _{\mathbf{u}_{i}} \sum_{t \in T}\left[u_{i, t} \cdot\left(\min _{\mathbf{p}_{i}}\left(c_{i, t}-\lambda_{D_{t}} \cdot p_{i, t}-\lambda_{R t} \cdot r_{i, t}\right)+s_{i, t}\right)\right]$

subject to constraints (4), and for hydro basin $b$

$L_{b}\left(\lambda_{D}, \lambda_{R}\right)=\min _{\mathbf{p}_{h}} \sum_{t \in T}-\left(\lambda_{D t} \cdot \sum_{h \in H_{b}} p_{h, t}+\lambda_{R t} \cdot \sum_{h \in H_{b}} r_{h, t}\right)$

subject to constraints (6).

Therefore, the LR approach decomposes the primal problem (1)-(6) into $|I|+|B|$ independent sub-problems, each one associated with a thermal unit and a hydro basin. In the solution of sub-problems (9) and (10), the values of $\lambda_{D}$ and $\boldsymbol{\lambda}_{R}$ are those as obtained from the current dual Lagrangian iteration. The solution method depends on the nature of the unit considered. In our UC formulation, the hydro sub-problem (10) is a linear model and any linear programming solver can be used. The inner minimization of thermal sub-problem (9) is a convex problem, which in our case permits closed-form solutions [2], while the outer is a mixed integer quadratic problem that is solved by means a forward dynamic programming (FDP), taking into account both the start-up prices and minimum up/down time constraints. Typically, the FDP requires a significant computational time. In [6] a procedure is suggested to reduce the number of states at each stage of the dynamic programming. Moreover, when Lagrangian multipliers of successive iterations are not too different, a significant speed improvement can be obtained by exploiting the $\mathbf{u}_{i}$ solution obtained at the previous Lagrangian iteration to compute an upper bound on the cost of the optimal solution and prune partial solutions with costs greater than the upper bound. This can be achieved if all the values associated to the nodes of the FDP are made non-negative, by subtracting their minimum value from each of them.

The optimal values $\lambda_{D}^{*}, \lambda_{R}^{*}$ of the Lagrange multipliers are obtained by the solution of the Lagrangian dual

$$
L^{*}=\max _{\lambda_{D}, \lambda_{R} \geq \mathbf{0}} L\left(\lambda_{R}, \lambda_{D}\right) \text {. }
$$

$L^{*}$ provides a lower bound on the optimal value of the objective function $C\left(\mathbf{u}, \mathbf{p}_{I}\right)$ of the primal problem (1)-(6) [28], and the difference between these two values is called (7) duality gap. As a by-product of the process of maximizing $L$, a schedule $\left\{\mathbf{u}, \mathbf{p}_{I}, \mathbf{p}_{H}\right\}$ is obtained from the solution of problem (7) with $\lambda_{D}=\lambda_{D}^{*}, \lambda_{R}=\lambda_{R}^{*}$. In general, this schedule does not satisfy constraints (2)-(3) and, therefore, techniques for computing a near-optimal schedule have to be implemented. These techniques are called Lagrangian heuristics.

The optimal value of the objective function of the primal problem will not be equal to $L^{*}$. At each iteration, by denoting with $L B$ the available highest value of $L$ and with $U B$ the 
available lowest value of $C\left(\mathbf{u}, \mathbf{p}_{I}\right)$, i.e. lower bound and upper bound of the sought optimal value respectively, the quality of the solution can be indicated by the relative duality gap $R D G=(U B-L B) / L B$. The smaller this value, the greater the quality of the available UC.

\section{B. Bundle methods for solving the Lagrangian dual}

Due to the problem formulation, the dual function $L\left(\boldsymbol{\lambda}_{D}, \boldsymbol{\lambda}_{R}\right)$ is non-differentiable, however it is concave and its sub-gradients with respect to the Lagrangian multipliers can be easily calculated: the $t^{\text {th }}$ element of the sub-gradient vector $\mathbf{g}_{D}\left(\lambda_{D}\right)$ with respect to $\lambda_{D}$ is

$g_{D t}\left(\boldsymbol{\lambda}_{D}\right)=D_{t}-\sum_{i \in I} u_{i, t} \cdot p_{i, t}-\sum_{h \in H} p_{h, t}$.

and the $t^{\text {th }}$ element of the sub-gradient vector $\mathbf{g}_{R}\left(\lambda_{R}\right)$ with respect to $\lambda_{R}$ is

$g_{R t}\left(\lambda_{R}\right)=R_{t}-\sum_{i \in I} u_{i, t} \cdot r_{i, t}-\sum_{h \in H} r_{h, t}$

In the following, we simplify the notation by denoting $\boldsymbol{\lambda}=\left[\boldsymbol{\lambda}_{D}, \boldsymbol{\lambda}_{R}\right], \mathbf{g}(\boldsymbol{\lambda})=\left[\mathbf{g}_{D}\left(\boldsymbol{\lambda}_{D}\right), \mathbf{g}_{R}\left(\boldsymbol{\lambda}_{R}\right)\right]$ and $\mathbf{G}=[\mathbf{D}, \mathbf{R}]$.

At iteration $k$ of a Bundle method, the dual function has been evaluated at $k$ multiplier vectors $\lambda^{0} \ldots \lambda^{k-1}$, and the corresponding values of the dual function, $L\left(\lambda^{0}\right) \ldots L\left(\lambda^{k-1}\right)$, and of the subgradients, $\mathbf{g}\left(\lambda^{0}\right) \ldots \mathbf{g}\left(\lambda^{k-1}\right)$, have been stored to form a bundle, denoted with $\beta$. The bundle is used to construct an upper approximation of $L(\lambda)$, the cutting plane (CP) model

$L_{\mathrm{CP}}^{k}(\lambda)=\min _{j \in \beta}\left\{L\left(\lambda^{j}\right)+\mathbf{g}\left(\lambda^{j}\right) \cdot\left(\lambda-\lambda^{j}\right)^{\prime}\right\}$.

where the prime indicates transpose. This approximation is tight at least in every point $\lambda^{j}$.

Now, the idea would be to maximize the known function $L_{\mathrm{CP}}$ instead of the unknown function $L$ and to use resultant vector $\lambda^{k}$ as the next iterate. A major drawback of this approach is that $L^{\mathrm{CP}}$ may be unbounded above, especially in the first iterations. Moreover, $L^{\mathrm{CP}}$ will be a poor approximation of $L$ if $\lambda^{k}$ is "too far" from the points $\lambda^{j}$. In order to overcome these drawbacks, we follow the so-called proximal Bundle method [26], namely a current point $\bar{\lambda}$ is selected (typically the one in the bundle that provides the greatest value of $L$ ), and the following quadratic problem is solved to find $\lambda^{k}$

$$
\max _{\lambda^{k}, v^{k}}\left\{v^{k}-\frac{1}{2 \cdot \alpha^{k}}\left\|\lambda^{k}-\bar{\lambda}\right\|^{2}\right\}
$$

subject to

$$
v^{k} \leq \delta^{j}+\mathbf{g}\left(\lambda^{j}\right) \cdot\left(\lambda^{k}-\bar{\lambda}\right)^{\prime} \quad \forall j \in \beta
$$

where $\quad \delta^{j}=L\left(\lambda^{j}\right)+\mathbf{g}\left(\lambda^{j}\right) \cdot\left(\bar{\lambda}-\lambda^{j}\right)^{\prime}-L(\bar{\lambda}) \quad$ (known as

linearization error), \|\|$^{2}$ is the Euclidean norm, and $\alpha_{k}$ is a positive parameter called trust-region parameter, as it suggests how far from $\bar{\lambda}$, model $L_{\mathrm{CP}}$ can be accepted as an approximation of $L$. This is illustrated by Fig. 1: practically, a quadratic penalty term has been added to $L_{\mathrm{CP}}$ to discourage choosing $\lambda^{k}$ far from $\bar{\lambda}$.

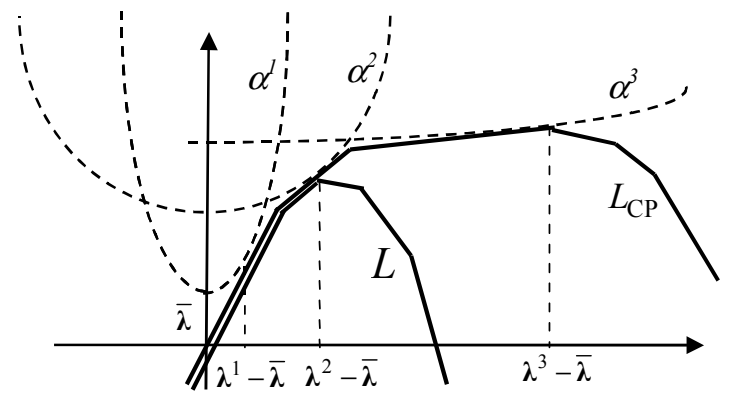

Fig. 1. Impact of parameter $\alpha: \lambda^{1}, \lambda^{2}, \lambda^{3}$ represent the solutions of problem (15)-(16) for three different values of $\alpha\left(\alpha^{1}<\alpha^{2}<\alpha^{3}\right)$.

Several important issues must be addressed in order to implement an efficient Bundle algorithm: among these, the dynamic choice of parameter $\alpha$ at each iteration, the rules for updating current point $\bar{\lambda}$, and the stopping criteria for the iterative algorithm. The procedure successfully proposed in [29] for the solution of Multicommodity Flow problems has been applied here to deal with these issues: at each iteration, the predicted increase $v^{k}$ is compared with the obtained increase $\Delta L^{k}=L\left(\lambda^{k}\right)-L(\bar{\lambda})$ and the current point is moved to $\lambda^{k}$ only if a sufficient increase has been obtained. If $\delta^{k}$ is "small", then the addition of $\mathbf{g}$ to the bundle is expected to consistently improve the accuracy of the $\mathrm{CP}$ model, otherwise $\alpha$ is decreased, forcing the next tentative point to be closer to $\bar{\lambda}$, where the $\mathrm{CP}$ model is hopefully accurate enough. The resulting algorithm is very stable and no special tuning for the update of the $\alpha$ parameter has been required.

Since the dual function $L(\lambda)$ exhibits a disaggregated structure, disaggregated Bundle approaches have been proposed for solving the Lagrangian dual $[12,16]$. The aggregated CP model $L_{\mathrm{CP}}^{k}(\lambda)$ is replaced with the sum of $|I|+|B|$ CP models, one for each thermal unit $i$ and river basin $b$. Equation (15) is replaced by

$\max _{\lambda^{k}, v_{I}^{k}, v_{B}^{k}}\left\{\sum_{i \in I} v_{i}^{k}+\sum_{b \in B} v_{b}^{k}-\frac{1}{2 \cdot \alpha^{k}}\left\|\lambda^{k}-\bar{\lambda}\right\|^{2}+\lambda \cdot \mathbf{G}^{\prime}\right\}$

subject to

$v_{i}^{k} \leq \delta_{i}^{j}+\mathbf{g}_{i}\left(\lambda^{j}\right) \cdot\left(\lambda^{k}-\bar{\lambda}\right)^{\prime} \quad \forall j \in \beta, \forall i \in I$

$v_{b}^{k} \leq \delta_{b}^{j}+\mathbf{g}_{b}\left(\lambda^{j}\right) \cdot\left(\lambda^{k}-\bar{\lambda}\right)^{\prime}$

$\forall j \in \beta, \forall b \in B$

where vectors $\mathbf{g}_{i}$ and $\mathbf{g}_{b}$ are the subgradients of $L_{i}$ and $L_{b}$ respectively, i.e. from Eq. (9) and (10) $g_{i, t}(\lambda)=\left[-p_{i, t},-r_{i, t}\right]$

and $g_{b, t}(\boldsymbol{\lambda})=\left[-\sum_{h \in H_{b}} p_{h, t},-\sum_{h \in H_{b}} r_{h, t}\right]$, and

$\delta_{i}^{j}=L_{i}\left(\lambda^{j}\right)+\mathbf{g}_{i}\left(\lambda^{j}\right) \cdot\left(\bar{\lambda}-\lambda^{j}\right)^{\prime}-L_{i}(\bar{\lambda})$

$\delta_{b}^{j}=L_{b}\left(\lambda^{j}\right)+\mathbf{g}_{b}\left(\lambda^{j}\right) \cdot\left(\bar{\lambda}-\lambda^{j}\right)^{\prime}-L_{b}(\bar{\lambda})$ 
The basic structure of the disaggregated algorithm remains similar to the structure of the aggregated one. It must be noted that each iteration in the solution of (17)-(19) is heavier than in (15); on the other hand, the sum of $|I|+|B|$ CP models is a much better description of $L(\boldsymbol{\lambda})$ than the aggregated CP model. Therefore, the disaggregated Bundle method is convenient if, as in our case (see the numerical results of Section VI), the longer computational time needed for each iteration is compensated by the strong reduction of the number of iterations required to converge to optimality [16].

\section{Lagrangian heuristics for the UC of thermal units}

As the solution of the dual problem does not produce a primal feasible solution, Lagrangian heuristics have to be implemented in order to modify this solution into a feasible UC.

In the literature (e.g. [4,5]), several heuristics have been proposed that can be applied independently of the method used to compute the solution of the dual problem. The output of these heuristics is a feasible matrix $\mathbf{u}^{*}$, which satisfies global constraints (2)-(3). A solution of the primal problem, (1)-(6), is computed by solving, for the given $\mathbf{u}^{*}$, the Economic Dispatch Problem (EDP), which is a convex quadratic problem in the considered UC formulation.

However, in some cases, the feasible schedules obtained by adopting these techniques may significantly differ from the optimal solution. To overcome this issue, in [22] integer programming techniques are developed for refining the UC solution obtained from solving the dual problem, by using the schedules generated by the different iterations of the Lagrangian method.

In the proposed approach, the use of such a refining yet complex procedure is avoided by the adoption of a Bundle method for solving the dual problem, which is characterized by high-quality convergence characteristics and provides also valuable information for generating a nearly optimal feasible solution to the primal problem. By suitably exploiting such information by the Lagrangian heuristic, as described in the following, the quality of the obtained UC may not need further refinement.

It is well known (e.g. [12,25,30]) that Bundle methods provide a way for solving, together with (11), also the following relaxed version of (1)-(6):

$\min _{\tilde{\mathbf{u}}, \tilde{\mathbf{s}}^{u}, \mathbf{p}_{I}} \sum_{i \in I} \sum_{t \in T}\left[\tilde{u}_{i, t} \cdot c_{i, t}\left(p_{i, t}\right)+\tilde{s}_{i, t}^{u}\right]$

subject to

$$
\begin{array}{ll}
\sum_{i \in I} \tilde{u}_{i, t} \cdot p_{i, t}+\sum_{h \in H} \tilde{p}_{h, t}=D_{t} & \forall t \in T \\
\sum_{i \in I} \tilde{u}_{i, t} \cdot r_{i, t}+\sum_{h \in H} \tilde{r}_{h, t} \geq R_{t} & \forall t \in T \\
\tilde{u}_{i, t} \cdot \underline{p}_{i} \leq p_{i, t} \leq \tilde{u}_{i, t} \cdot \bar{p}_{i} & \\
\left(\tilde{\mathbf{u}}_{i}, \tilde{\mathbf{s}}_{i}\right) \in \operatorname{conv}\left\{\left(\mathbf{u}_{i}, \mathbf{s}_{i}\right): \mathbf{u}_{i} \in U_{i}\right\} & \forall i \in I
\end{array}
$$

constraints equivalent to (6)

where $\operatorname{conv}\left\{\left(\mathbf{u}_{i}, \mathbf{s}_{i}\right): \mathbf{u}_{i} \in U_{i}\right\}$ indicates the convex hull of the set $U_{i}$ of commitment schedules $\mathbf{u}_{i}$ (and the corresponding start-up costs $\mathbf{s}_{i}$ ) that satisfy $\tau_{i}^{d}$ and $\tau_{i}^{u}$ constraints. In fact, at each iteration $k$ and $\forall j \in \beta$, nonnegative multipliers $\theta_{i}^{j}$ and $\theta_{b}^{j}$ can be found, at no extra cost, from the solution of (17)(19), such that $\sum_{j} \theta_{i}^{j}=1, \sum_{j} \theta_{b}^{j}=1$, and the "convexified solution" $\quad \tilde{u}_{i, t}=\sum_{j} \theta_{i}^{j} \cdot u_{i, t}^{j}, \quad \tilde{s}_{i, t}=\sum_{j} \theta_{i}^{j} \cdot s_{i, t}^{j} \quad$ and $\tilde{p}_{h, t}=\sum_{j} \theta_{b}^{j} \cdot p_{h, t}^{j}$, for $h \in H_{b}$, converge to an optimal solution of (20)-(21). In the aggregated case, at every iteration $k$, all the multipliers $\theta_{i}^{j}$ and $\theta_{b}^{j}, \forall i \in I$ and $\forall b \in B$, have the same value $\theta^{j}$ that can be found, at no computational cost, by the solution of (15)-(16).

The solution $\tilde{\mathbf{u}}$ does not, in general, satisfy constraints (2) and (3), except at the last Lagrangian iteration, but it typically becomes almost feasible with respect to these constraints very rapidly. Being it fractional (i.e. $\left.\tilde{u}_{i, t} \in[0,1]\right), \tilde{\mathbf{u}}$ is not a feasible schedule, but each element $\tilde{u}_{i, t}$ of the matrix can be interpreted as the "probability" for unit $i$ to be committed at period $t$. As shown in $[12,30]$, use of this matrix to define priority lists of the thermal units, in some different possible ways, turns out to produce good solutions. In our approach, we have extended this idea by making combined use of "probabilities" and costs: more specifically we use the ratio between $\tilde{u}_{i, t}$ and $\tilde{u}_{i, t} \cdot c_{i, t}\left(p_{i, t}\right)+\tilde{s}_{i, t}$ in order to define the priority list of the thermal units, used in the Lagrangian heuristic to find a feasible schedule. Also a shutdown heuristic has been implemented (as in [12]), trying to shutdown units whenever possible, using a reversed priority list. As shown in Section VI, this improves the overall performance of the algorithm and the quality of the obtained schedules.

The following section presents an extension of the above-described Lagrangian heuristic to the case of hydrothermal UC. Moreover, details of a warm-start procedure specific for the proposed approach will be given.

\section{LAGRANGIAN HEURISTIC FOR HYDROTHERMAL UC AND WARM-START PROCEDURE}

\section{A. Lagrangian heuristics for the hydrothermal case}

The LR heuristics described for thermal units cannot be directly applied to hydro units due to energy constraints (6) that couple the hydro unit schedules along the whole optimization horizon.

To find a feasible solution, an option is to fix the hydro schedule, as obtained from the current dual solution, subtracting it from the demand and reserve profiles, and then to apply the heuristics only to the thermal unit schedules. However this approach is, in general, inefficient. The main reason of failure can be illustrated by the following simple numerical example of UC of two thermal units, with linear cost functions, and two hydro units, located in different hydro valleys, over a two time periods with constant demand:

$\min _{\mathbf{u}, \mathbf{p}_{I}, \mathbf{p}_{H}} \sum_{t=1}^{2}\left\{u_{1, t} \cdot\left(10 p_{1, t}+100\right)+u_{2, t} \cdot\left(50 p_{2, t}+200\right)\right\}$ 
subject to

$$
\begin{array}{ll}
\sum_{i=1}^{2} p_{i, t}+\sum_{h=3}^{4} p_{h, t}=300 & t=1,2 \\
50 \leq p_{i, t} \leq 250 & i=1,2 \quad t=1,2 \\
w_{h, 1}+w_{h, 2} \leq 50 & h=3,4 \\
0 \leq p_{h, t}=w_{h, t} \leq 50 & h=3,4 \quad t=1,2
\end{array}
$$

For both hydro units $h=3$ and $h=4$, if $\lambda_{1} \neq \lambda_{2}$ the solutions of (10) subject to constraints (e4) are $p_{h, 1}=50$ and $p_{h, 2}=0$, if $\lambda_{1}>\lambda_{2}$; while they become $p_{h, 1}=0$ and $p_{h, 2}=50$, if $\lambda_{1}<\lambda_{2}$. Therefore, tiny differences in the multipliers determine two very different hydro schedules, due to the energy constraint. Using any one of these two solutions forces the commitment of the second and more expensive thermal unit, with $C_{2}\left(p_{2 \min }\right)>C_{1}\left(p_{1, \max }\right)$, for one period, while a less expensive hydro schedule exists that allocates the available energy equally in every hour and avoids the use of the second unit.

To solve this kind of oscillating behavior of the LR algorithm, Augmented Lagrangian approaches (e.g. [18]) and nonlinear approximation methods [23] have been proposed. A modified version of the heuristic presented in [5] is adopted in [24] to modify the hydro schedule.

In this paper we consider an alternative Lagrangian heuristic that avoids the modification of the linear model of hydro units. The idea is to use the convexified hydro schedule $\tilde{\mathbf{p}}_{H}$ as a replacement for schedule $\mathbf{p}_{H}$ given by the current dual solution, in order to evaluate the demand and reserve profiles that have to be met by thermal units. The LR heuristics based on the use of fractional matrix $\tilde{\mathbf{u}}$ is then applied to find a feasible thermal schedule.

The thermal part of the UC problem is strongly non-convex, on the contrary the considered hydro model is convex, besides being linear. Therefore, $\tilde{\mathbf{p}}_{H}$ is a feasible hydro schedule at each iteration, while, being fractional, $\tilde{\mathbf{u}}$ is not, in general, a feasible thermal schedule even at the last iteration. This shows the importance of using a disaggregated Bundle method, since it detaches the convex hydro component of the problem from the non-convex thermal one, leading to a zero gap solution for the former.

The heuristic procedure is applied to the dual solutions at each, but the first, Lagrangian iterations, since, as already observed (e.g. [4]), there is no guarantee that the best feasible $\mathrm{UC}$ is found from the optimal dual solution.

\section{B. Warm-starting procedure}

A warm-starting procedure is important to compute good initial estimates of Lagrangian multipliers $\lambda^{0}$, which are the starting point for the Bundle method. This improves the convergence of the algorithm by providing both a good solution of the Lagrangian dual and an upper-bound to the $\mathrm{CP}$ models.

The proposed warm-start procedure is based on the solution of a relaxed version of problem (1)-(6), where $\mathbf{u}$ is replaced by a matrix $\hat{\mathbf{u}}$, whose values are no longer integer variables (i.e. $\hat{u}_{i, t} \in[0,1]$ ), and constraints $\tau_{i}^{u}$ and $\tau_{i}^{d}$ are formulated in the following linear form, for each thermal unit $i$ and time period $t$ :

$$
\begin{array}{ll}
\hat{u}_{i, t+r} \geq \hat{u}_{i, t}-\hat{u}_{i, t-1} & \forall r=1 \ldots \min \left(\tau_{i}^{u}, T-t\right) \\
\hat{u}_{i, t+r} \leq 1-\hat{u}_{i, t-1}+\hat{u}_{i, t} & \forall r=1 \ldots \min \left(\tau_{i}^{d}, T-t\right)
\end{array}
$$

This problem is quadratic and convex, and its solution by an interior-point algorithm provides the dual optimal multipliers associated with coupling constraints (2) and (3) that are used as $\lambda_{D}^{0}$ and $\lambda_{R}^{0}$ respectively.

Moreover, the same warm-start procedure calculates the initial values of subgradients $\mathbf{g}_{i}\left(\lambda^{0}\right)$ and $\mathbf{g}_{b}\left(\lambda^{0}\right)$, of (18) and (19), which are added to the bundle to build the CP models at the following iterations. However, for the calculation of $\mathbf{g}_{i}\left(\lambda^{0}\right)$, matrix $\hat{\mathbf{u}}$ must be replaced by a matrix of type $\mathbf{u}$, feasible for the Lagrangian dual, i.e. with binary values that satisfy also the original $\tau_{i}^{u}$ and $\tau_{i}^{d}$ constraints. For this purpose, in the proposed warm-start procedure a priority-list heuristic (as in [4]) has been applied. Since the hydro model is linear, the hydro schedule evaluated by the above-mentioned relaxed version of the primal problem is already feasible and can be directly used to calculate $\mathbf{g}_{b}\left(\lambda^{0}\right)$. The efficiency of the warm-starting procedure is manly due to this aid in the construction of the bundle used by the CP models at the following iterations for the solution of the Lagrangian dual. The CP models have also an impact on the behavior of the proposed LR heuristics, since, as already described, they provide the convexified solutions $\tilde{\mathbf{u}}$ and $\tilde{\mathbf{p}}_{H}$, starting points for the LR heuristic.

Also, the impact of the proposed warm-start procedure is enhanced by the adoption of the Bundle method in a disaggregated form. In fact, as already mentioned, since the hydro schedule calculated by the warm-start procedure is given directly by the solution of the proposed relaxed version of the primal problem, in general, it results a very good estimate of $\tilde{\mathbf{p}}_{H}$. Conversely, as it will be illustrated in the next section, the impact of the proposed warm start procedure is very limited if an aggregated Bundle method is adopted. 


\section{NUMERICAL RESULTS}

\section{A. Implementation of the algorithms}

This section presents some results obtained by a prototype version of the code that was implemented in AMPL [31], an algebraic modeling language for mathematical programming. A major advantage is the availability of a set of drivers that allows the use of solvers for the linear and quadratic problems of the proposed LR approach, i.e. (10), (17), the relaxed version of the primal problem in the warm-start procedure and the EDP (primal problem (1)-(6) with matrix u known as computed by the heuristic procedure). Several solvers have been successfully used (CPLEX 6.0 [32], LOQO 4.05 [33] and MOSEK 2.0 [34] $)^{3}$

\section{B. Instances description.}

The code has been used to perform a number of studies to assess the characteristics of the proposed approach. The data of the various hydrothermal systems are generated, starting from various test cases proposed in the literature, by a statistical procedure described in [35]. This procedure allows assessing the efficiency of the algorithm under different, reasonable, conditions. Instances have been considered with a small or large number of units, with prevalence of units of large size or of small size, with different percentages of hydro production, with thermal units characterized by similar or very different costs. Also the influence of the load demand can be analyzed, by generating high and low demands as well as flat or bumpy load profiles.

In the following, the results obtained with the proposed LR approach relevant to a test case with 45 thermal units and 20 hydro units on a 24-hours horizon are presented. The considered load profile is shown in Fig. $4^{4}$. The results are also compared with those obtained by using different methods for the solution of the Lagrangian dual and different Lagrangian heuristics. Similar behaviors of the algorithms have been observed for all the considered instances.

\section{Comparative results and analysis}

The analysis regards: 1) the convergence characteristics of different methods in the solution of the Lagrangian dual and 2) the impact of Lagrangian heuristics for the modification of the dual solution in a feasible UC.

\section{1) Convergence of the Lagrangian dual solution}

Fig. 2 presents the values of dual function $L$, at the various iterations, obtained by using the proposed approach, based on disaggregated Bundle method with the proposed Lagrangian heuristic and warm-start procedure, and four algorithms that implements the following methods (all methods, but method 4 , use, as starting point, the values of multipliers $\lambda^{0}$ evaluated by the warm-start procedure):

d1 subgradient method (as in [4]);

d2 aggregated Bundle method;

d3 proposed approach, without including in the bundle the values of $\mathbf{g}\left(\lambda^{0}\right)$ calculated by the warm-start procedure; d4 disaggregated Bundle method as method $\mathrm{d} 3$, but starting with all the multipliers $\lambda^{0}$ set to zero.

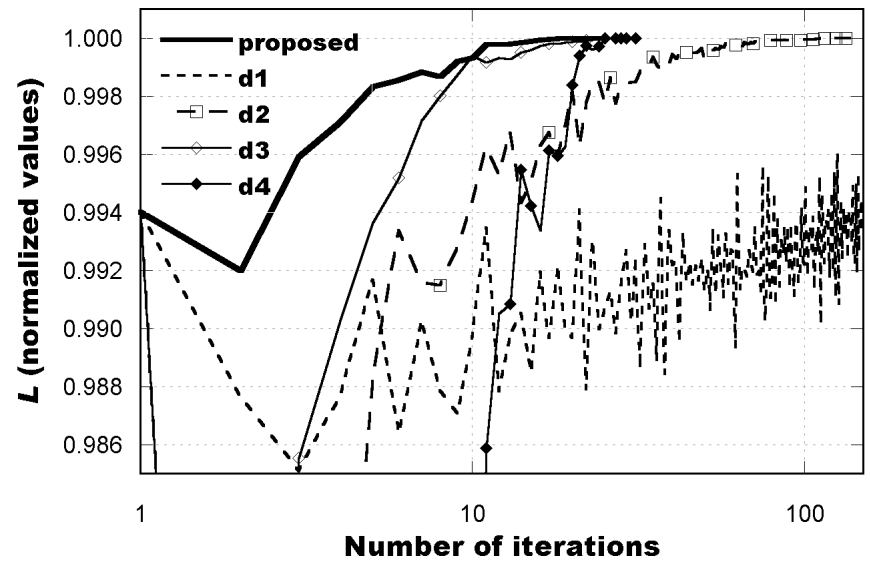

Fig. 2. Behavior of the dual function $L$ through the Lagrangian iterations, as computed by using the proposed approach and four other methods (d1, d2, d3 and d4) described in the text. Normalized values are obtained by dividing the values of $L$ by the obtained maximum value.

Fig. 2 shows that, although Bundle methods are typically not ascent approaches, i.e., improvement in the dual value at each iteration in general cannot be guaranteed, the proposed warm-start procedure makes the Bundle method to behave essentially as an ascent algorithm. Thus, the warm-start procedure avoids the typical large fluctuations in the dual function values and, as early theoretically described, improves the convergence speed. Avoiding large fluctuations is beneficial also to the speed of the FDP algorithm, as mentioned in paragraph IV. As expected, the number of iterations obtained with disaggregated Bundle methods is always lower than that obtained with the aggregated one.

\section{2) Impact of the Lagrangian heuristics}

Fig. 3 presents the values of the objective function of the primal problem (1), at the various iterations, obtained using the proposed approach and four algorithms that implements the following methods (all using, as starting point, the values of multipliers $\lambda^{0}$ evaluated by the warm-start procedure and, for the thermal units, the Lagrangian heuristic based on the convexified matrix $\tilde{\mathbf{u}}$ ):

p1 no modification of the hydro schedule obtained from the dual solution given by an aggregate Bundle method;

p2 proposed Lagrangian heuristic and aggregate Bundle method to obtain the dual solution;

p3 no modification of the hydro schedule obtained from the dual solution given by a disaggregate Bundle method;

p4 proposed approach, without including in the bundle the values of $\mathbf{g}\left(\lambda^{0}\right)$ calculated by the warm-start procedure.

\footnotetext{
${ }^{3} \mathrm{~A} \mathrm{C}++$ object oriented version of the code, which implements the same proposed procedure, is currently under development. While in the prototype version of the code, each basin is assumed to contain exactly one unit, this constraint is relaxed in the new version.

${ }^{4}$ The complete set of input data is available from the authors.
} 


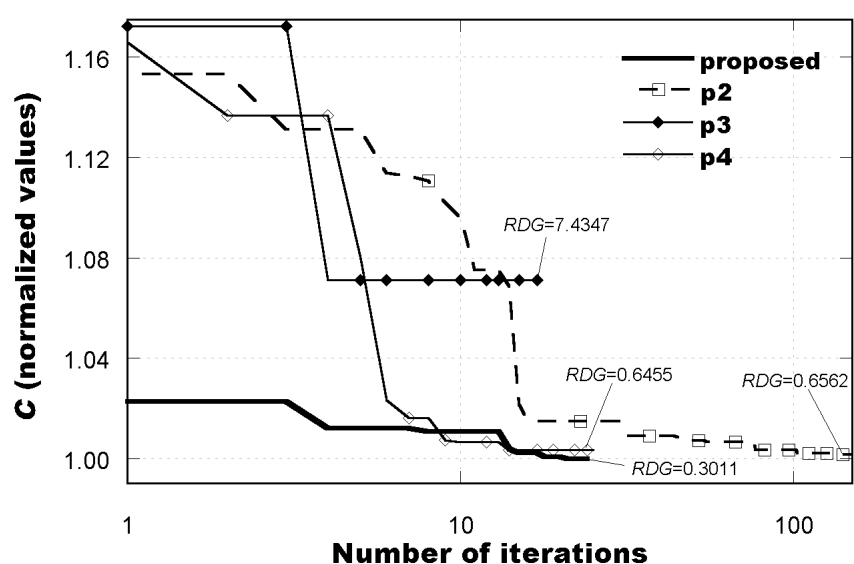

Fig. 3 Behavior of the objective function $C$ through the Lagrangian iterations, as computed by using the proposed approach and others methods (p2, p3 and p4) described in the text. Normalized values are obtained by dividing the values of $C$ by the obtained minimum value.

Fig. 3 does not show the results of method p1, because of its failure in reaching a solution. Indeed we have observed that by using the current hydro schedule as given by the solution of the Lagrangian dual, in many cases a feasible solution cannot be reached. The methods $\mathrm{p} 2$ and $\mathrm{p} 4$ give practically the same final result, but the disaggregate approach requires a lower number of iterations. Fig. 3 shows also the impact of the proposed warm-start procedure that provides at the first iteration a primal solution only about $2.3 \%$ greater than the final result. Moreover, the inclusion in the bundle of the initial subgradients, evaluated by the warm-start procedure, improves both the convergence characteristics and the final result of the proposed approach. We can observe that this combination improves its primal values practically at each iteration and ours calculations have shown that this is a typical behavior for most of the considered instances.

Unlike classical subgradient algorithms, Bundle methods possess effective stopping criteria that allow to prove that the current solution is optimal within a specified tolerance, and therefore to terminate the search. An alternative stopping criterion is to stop when $R D G$ reaches a desired value; however, since the obtained $R D G$ depends on both the dual optimization process and the Lagrangian heuristic, as well as on the inherent duality gap, we used the standard stopping criteria of the Bundle method with a tolerance equal to $1 \mathrm{e}^{-6}$. If a stopping criterion of $R D G<1 \%$ is used, the solution is usually found in four or five iterations.

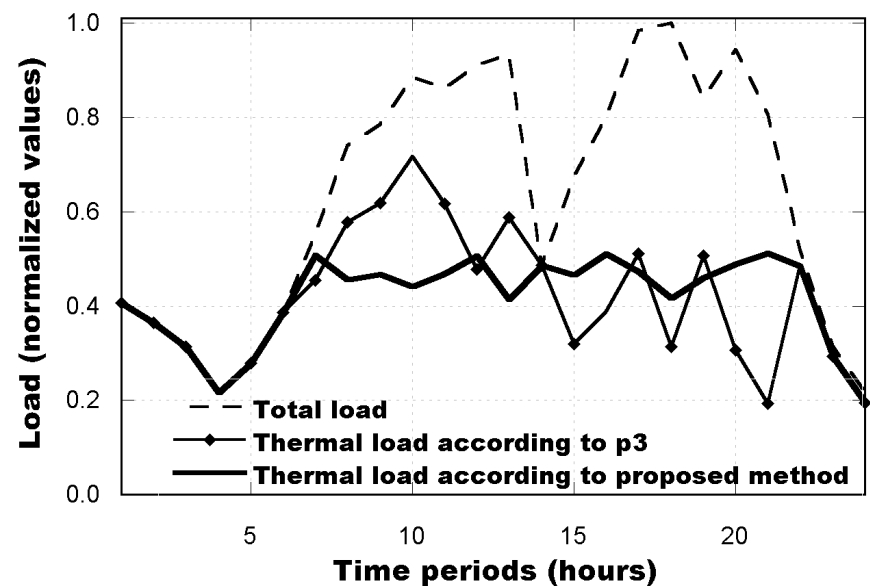

Fig. 4. Total load profile (dashed lines) and part covered by thermal production, as calculated by using the proposed modification of the dual hydro schedule (thick solid line) and without modification, i.e., p3 method, (thin solid line). Normalized values are obtained by dividing the load values by the maximum value.

Fig. 4 shows the load profile together with the part of the load that has to be covered by the production of the thermal units, obtained in the best UC of Fig. 2 found by the proposed method and by method $\mathrm{p} 3$. This figure shows that using the proposed Lagrangian heuristic for the hydro schedule the thermal units have to satisfy a flatter load profile. This allows the thermal generation park working at lower marginal costs.

Table I summarizes the results obtained in two other cases: case 1 with 75 thermal and 45 hydro units, and case 2 with 105 thermal and 75 hydro units, over a 24-hours horizon. The best $R D G$ values and the number of iterations required to reach them are reported for the proposed approach and for the other methods. The impact of the proposed heuristic for the modification of the dual hydro schedule is also shown. A maximum number of iterations equal to 150 has been set.

With all the methods used for the solution of the Lagrangian dual, the computation of the Lagrangian function takes more than $90 \%$ of the computational time; therefore, the total time is almost linear with the number of iterations. For the considered cases, the average computational time required by each iteration of the disaggregate Bundle method is only about 3-4\% larger than that of the aggregated Bundle method. Such an increase is largely compensated by the strong reduction of the number of the required iterations.

As shown in Table I, without applying the proposed

TABLE I - BEST $R D G$ VALUES AND NUMBER OF ITERATIONS REQUIRED TO REACH THEM

\begin{tabular}{|c|c|c|c|c|c|c|c|}
\hline \multicolumn{4}{|c|}{ Case 1 } & \multicolumn{4}{c|}{ Case 2 } \\
\hline $\begin{array}{c}\text { Method for } \\
\text { the dual }\end{array}$ & $\begin{array}{c}\text { Modification of the dual } \\
\text { hydro schedule* }\end{array}$ & $\begin{array}{c}R D G \\
\%\end{array}$ & $\begin{array}{c}\text { No. of } \\
\text { iterations }\end{array}$ & $\begin{array}{c}\text { Method for } \\
\text { the dual }\end{array}$ & $\begin{array}{c}\text { Modification of the dual } \\
\text { hydro schedule* }\end{array}$ & $\begin{array}{c}R D G \\
\%\end{array}$ & $\begin{array}{c}\text { No. of } \\
\text { iterations }\end{array}$ \\
\hline $\mathrm{d} 1$ & $\mathrm{no}$ & $\mathrm{FAIL}$ & 150 & $\mathrm{~d} 1$ & no & 5.3657 & 150 \\
\hline $\mathrm{d} 2$ & $\mathrm{no}$ & 4.3105 & 143 & $\mathrm{~d} 2$ & no & 4.3105 & 148 \\
\hline $\mathrm{d} 3$ & $\mathrm{no}$ & 4.5275 & 21 & $\mathrm{~d} 3$ & no & 4.5275 & 21 \\
\hline $\mathrm{d} 2$ & Yes & 0.5403 & 150 & $\mathrm{~d} 2$ & yes & 0.4544 & 150 \\
\hline $\mathrm{d} 3$ & Yes & 0.5234 & 26 & $\mathrm{~d} 3$ & yes & 0.4556 & 24 \\
\hline $\mathrm{d} 4$ & Yes & 0.5648 & 39 & $\mathrm{~d} 4$ & yes & 0.4534 & 41 \\
\hline \multicolumn{2}{|c|}{ Proposed approach } & 0.3895 & 24 & \multicolumn{2}{c|}{ Proposed approach } & 0.2454 & 23 \\
\hline
\end{tabular}

* as described in Section V.A. 
warm-start procedure and if the hydro schedule is not modified as described in Section V.A, the disaggregated Bundle method gives solutions of quality often lower than the aggregate one. This result is in agreement with those presented in [30]. The adoption of the proposed Lagrangian heuristic to modify the hydro schedule leads to a substantial improvement in the quality of the primal solutions; in this case disaggregated Bundle methods prove more efficiency than aggregate ones both in the convergence characteristics and in the quality of the primal solution. This fact is enhanced by the adoption of the proposed warm-start procedure.

\section{CONCLUSIONS}

In this paper, a Lagrangian Relaxation approach for the solution of hydrothermal UC problems has been presented. The proposed approach solves the dual problem through a disaggregated Bundle method; the feasible solution for the primal problem is computed by a heuristic procedure that exploits available information given by the Bundle algorithm.

Some problems arising in solving the dual problem in presence of linearly modeled components such as hydro units have been described in the paper, which have been solved by using a convexified hydro schedule generated at each iteration by the Bundle method. This greatly improved the quality of the primal solutions found.

A relaxation of the primal problem is also proposed to be used to warm-start the Bundle algorithm. This makes the Bundle method to behave essentially as an ascent algorithm, avoiding the typical large fluctuations in the dual function values and therefore being beneficial to the convergence characteristics, to the quality of the feasible primal solutions generated by the Lagrangian heuristic and to the speed of the dynamic programming required to handle the operational constraints of the thermal units.

The proposed approach has been applied to a hydrothermal UC problem, assuming a linear hydro system model; clearly, additional research is needed to extend the proposed Lagrangian heuristic to more complex hydro system representations.

The overall results obtained by the implemented Lagrangian relaxation approach are of very good quality and they are reached within few iterations. We feel that the proposed method could be of help for the solution of UC of hydrothermal power generation systems in the uncertain environment of the competitive electricity markets.

Also, although we have performed our experiments with a proximal Bundle method and for a relatively simple version of the UC problem, the results are expected to be of interest even for similar Lagrangian optimization procedures (e.g. [7,8,17]), and more complex versions of the UC problem.

\section{REFERENCES}

[1] B. F. Hobbs, M. Rothkopf, R. P. O'Neill and H. P. Chao (eds.), The next generation of unit commitment models, Boston, Kluwer Academic Press, 2001.

[2] A. J. Wood and B. F. Wollemberg, Power generation operation and control, John Wiley \& Sons, 1996.
[3] A. Merlin and P. Sandrin, "A new method for unit commitment at Electricité de France", IEEE Trans. Power Apparatus and Systems, PAS-102, no. 5, pp. 1218-1225, 1983.

[4] J. F. Bard, "Short-term scheduling of thermal-electric generators using Lagrangian Relaxation", Operations Research, vol. 36, pp. 756-766, 1988.

[5] F. Zhuang and F. D. Galiana, "Towards a more rigorous and practical unit commitment by Lagrangian Relaxation", IEEE Trans. Power Systems, vol. 3, pp. 763-773, 1988.

[6] S. Takriti, J.R. Birge and E. Long, "A stochastic model for unit commitment", IEEE Trans. Power Systems, vol. 11, no. 3, pp. 1497$1508,1996$.

[7] N. Jiménez Redondo and A. J. Conejo, "Short-term hydro-thermal coordination by Lagrangian Relaxation-solution of the dual problem", IEEE Trans. Power Systems, vol. 14, n. 1, pp. 89-95, 1999.

[8] M. Madrigal and V. H. Quintana, "An Interior-Point/Cutting-Plane method to solve unit commitment problems", IEEE Trans. Power Systems, vol. 15, no. 3, pp. 1022-1027, 2000.

[9] C. Lemaréchal, F. Pellegrino, A. Renaud and C. Sagastizábal, "Bundle methods applied to the unit commitment problem", in Proc. the 17th IFIP-Conference on System Modelling and Optimization, Prague, July 10-14, 1995.

[10] A. Möller and W. Römisch, "A Dual method for the unit commitment problem", Humboldt-Universität Berlin, Institut für Mathematik, Preprint, no. 95-1, 1995.

[11] F. Pellegrino, A. Renaud and Th. Socroun, "Bundle and Augmented Lagrangian methods for short-term unit commitment", in Proc. 12th Power Systems Computation Conference, Dresden, 1996.

[12] S. Feltenmark, "On optimization of power production", Ph.D. Thesis, Dept. of Mathematics/Optimization, Royal Institute of Technology, Stockholm, Sweden, 1997.

[13] R. Gollmer, A. Möller, M. P. Nowak, W. Römisch and R. Schultz, "Primal and Dual methods for unit commitment in a hydro-thermal power system", in Proc. 13th Power Systems Computation Conference, Trondheim, Norway, 1999.

[14] Shih-Yih Lai and R. Baldick, "Unit commitment with ramp multipliers", IEEE Trans. Power Systems, vol. 14, no. 1, pp. 58-64, 1999

[15] D. Zhang, P. B. Luh and Y. Zhang, "A Bundle method for hydrothermal scheduling", IEEE Trans. Power Systems, vol. 14, no. 4, pp. 1355-1361, 1999.

[16] L. Bacaud, C. Lemaréchal, A. Renaud and C. Sagastizábal, "Bundle methods in stochastic optimal power management: a disaggregated approach using preconditioners", Computational Optimization and Applications, vol. 20, pp. 227-244, 2001.

[17] P. B. Luh, D. Zhang and R.N. Tomastik, "An algorithm for solving the dual problem of hydrothermal scheduling", IEEE Trans. Power Systems, vol. 13, n. 2, pp. 593-600, 1998.

[18] J. Batut and A. Renaud, "Daily generation scheduling optimization with transmission constraints: a new class of algorithms" IEEE Trans. Power System, vol. 7, no. 3, pp. 982-989, 1992

[19] S. J. Wang, S.M. Shahidehpour, D. S. Kirschen, S. Mokhtari and G. D. Irisarri, "Short-term generation scheduling with transmission and environmental constraints using an Augmented Lagrangian Relaxation", IEEE Trans. Power Systems, vol. 10, no. 3, pp. 1294-1301, 1995.

[20] S. Al-Agtash and R. Su, "Augmented Lagrangian approach to hydrothermal scheduling", IEEE Trans. Power Systems, vol. 13, no. 4, pp. 1392-1400, 1998.

[21] C. Beltran Royo, "Generalized unit commitment by the Radar Multiplier method", Ph.D. dissertation, Universitat Politècnica de Catalunya, 2001.

[22] S. Takriti and J. R. Birge, "Using Integer programming to refine Lagrangian-based unit commitment solutions", IEEE Trans. Power Systems, vol. 15, no. 1, pp. 151-156, 2000.

[23] X. Guan, P. B. Luh and L. Zhang, "Non linear approximation method in Lagrangian Relaxation-based algorithms for hydrothermal scheduling", IEEE Trans. Power Systems, vol. 10, n 2, pp. 772-778, 1995.

[24] N. Gröwe-Kuska, K.C. Kiwiel, M. P. Nowak, W. Römisch and I. Wegner, "Power management under uncertainty by Lagrangian Relaxation", in Proc. 6th International Conference Probabilistic Methods Applied to Power Systems, PMAPS, Porto, 2000.

[25] A. Frangioni, "Generalized Bundle methods", SIAM Journal of Optimization, to be published. 
[26] J-B. Hiriart-Urruty and C. Lemaréchal, Convex analysis and minimization algorithms II - Advanced theory and Bundle methods, A Series of Comprehensive Studies in Mathematics, vol. 306, SpringerVerlag, 1993

[27] P. Sandrin et al., "Unit commitment", CIGRE SC38 TF38-04-01 Final Report, 1997.

[28] A. Geoffrion, "Lagrangian relaxation for integer programming", Mathematical programming study, vol. 2, pp. 82-114, 1974.

[29] A. Frangioni and G. Gallo, "A Bundle type dual-ascent approach to linear multicommodity min cost flow problems", INFORMS Journal on Computing, vol. 11, no. 4, p. 370-393, 1999.

[30] S. Feltenmark and K.C. Kiwiel, "Dual applications of proximal Bundle methods including Lagrangian Relaxation of nonconvex problems", SIAM Journal of Optimization, vol. 10, no. 3, pp 697-721, 2000.

[31] R. Fourer, D.M. Gay and B.W. Kernighan, AMPL: a modeling language for mathematical programming, Scientific Press, 1993.

[32] R. E. Bixby, S. Ceria, C. M. McZeal and M. W. P. Savelsbergh, "An updated mixed integer programming library: MIPLIB 3.0", Optima, vol. 54, pp. 12-15, 1998.

[33] R. J. Vanderbei, "LOQO: An Interior Point code for quadratic programming", Optimization Methods and Software, vol. 12, pp. 451484, 1999.

[34] E. D. Andersen and K. D. Andersen, "The MOSEK Interior Point optimizer for linear programming: an implementation of the Homogeneous algorithm", in High Performance Optimization, $\mathrm{H}$. Frenk, K. Roos, T. Terlaky, and S. Zhang (eds.), pp. 197-232, Kluwer Academic Publishers, 2000.

[35] A. Borghetti, A. Frangioni, F. Lacalandra, A. Lodi, S. Martello, C. A. Nucci and A. Trebbi, "Lagrangian Relaxation and Tabu Search approaches for the unit commitment problem", in Proc. IEEE Porto Power Tech Conference, Porto, Portugal, September 10-13, 2001.

[36] W. Fan, X. Guan and Q. Zhai, "A new method for unit commitment with ramping constraints", Electric Power Systems Research, vol. 62, pp. 215-224, 2002.
Alberto Borghetti graduated with honors in Electrical Engineering from the University of Bologna, Italy, in 1992. Since then he has been working with the power system group at the same University, where he was appointed University Researcher in Electric Power Systems in 1994. He is author or co-author of about 30 scientific papers presented at international conferences or published on reviewed journals. His main research interests are power system simulation, with reference to voltage collapse, system restoration, electromagnetic transients due to lightning and generation scheduling.

Antonio Frangioni graduated in Computer Science from the University of Pisa, Italy, in 1992, and got his Ph.D. in Computer Science from the same University in 1996. Since December 1996 he is Research Associate at the Computer Science Department of the University of Pisa, within the Operations Research Group. His main research interests are in continuous and combinatorial optimization, especially decomposition algorithms for largescale optimization problems, for which he has developed and implemented several efficient solvers, both sequential and parallel. He is author and coauthor of about 10 publications among journal papers and book articles, plus some conference presentations.

Fabrizio Lacalandra is a student at the Department of Electrical Engineering of the University of Bologna, Italy, working with the power system group at the same University.

Carlo Alberto Nucci was born in Bologna, Italy, in 1956. Received a de-gree with honors in electrical engineering in 1982 from the University of Bolo-gna. Joined the same university in 1983 as Researcher in the Power Elec-trical Engineering Institute. Associate professor in the same University in 1992, full professor in Power Systems in 2000. His research interests concern power systems transients, with particular reference to lightning and nuclear EMP impact on power lines, power systems dynamics, unit commitment, and the study of power components including medium voltage capacitors and traction batteries. He is the responsible member of the Task Force 33.01.01 "Lightning-induced overvoltages" of the CIGRE Working Group 33.01 "Lightning", secretary of the same Working Group, member of the IEEE Working Group on the Lightning Performances of Distribution Lines, and member of the CIGRE Working Group 36.07 "Power quality indices and objectives". He is author or coauthor of more than 130 scientific papers presented at international conferences and published on reviewed journals. 